\title{
阿秒光源前沿科学与应用
}

付玉喜 1,2

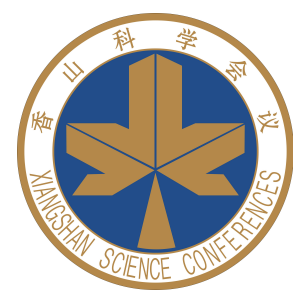

1. 中国科学院西安光学精密机械研究所, 西安 710119;

2. 中国科学院大学光电学院, 北京 100049

E-mail: fuyuxi@opt.ac.cn

\section{Frontiers in science and applications for attosecond light source}

\author{
Yuxi $\mathrm{Fu}^{1,2}$ \\ ${ }^{1}$ Xi'an Institute of Optics and Precision Mechanics, Chinese Academy of Sciences, Xi'an 710119, China; \\ ${ }^{2}$ School of Optoelectronics, University of Chinese Academy of Sciences, Beijing 100049, China \\ E-mail: fuyuxi@opt.ac.cn
}

doi: 10.1360/TB-2020-1672

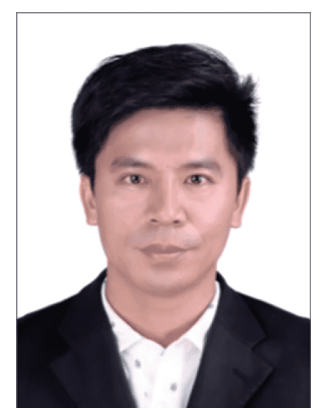

\section{付至喜}

中国科学院西安光学精密机械 研究所研究员, 博士生导师, 日 本理化学研究所客座研究员. 从事高能量中红外飞秒激光技 术、阿秒科学与技术、非线性 光学等研究, 在高能量中红外飞 秒激光和小型化软 X射线阿秒光 源研制方面取得了重要突破。荣 获第2届RIKEN Baiho Award和 第13届大阪大学Kondo Prize. 2020 年获得陕西省和中国科学 院高层次人才项目支持.
2001 年, 科学家首次在实验中产生了阿秒 $\left(10^{-18} \mathrm{~s}\right)$ 量级的超短光脉冲 ${ }^{[1]}$, 从此 打开了阿秒科学的大门. 迄今为止, 阿秒光源是人类所能掌握的唯一同时具有纳 米空间分辨率和阿秒时间分辩率的全相千光源. 由于其重大的科学意义和广泛 的应用领域，部分欧洲国家和美国、日本、韩国等相继制定了阿秒光源研究计 划, 如美国将阿秒光源产生技术列入 21 世纪的20项战略科学技术之一; 日本将其 列入6大核心技术之一; 德国视之为优先发展技术, 并成立了国际马普阿秒科学 中心. 经过近20年的发展, 阿秒光源的产生和应用技术逐渐成熟, 并已经在原子 分子物理、化学等基础学科领域取得了诸多突破性研究成果, 目前正快速向凝 聚态物理、材料科学、生命科学和医学等更具实用价值的领域拓展. 作为一种 先进的高精尖极端条件实验平台, 阿秒光源大科学装置的建设被许多国家提上 日程并付诸实施. 由欧盟主导的欧洲极端光设施-阿秒光源(ELI-ALPS) 已经初步 建成.

正是由于阿秒科学的重要意义, 我国也有众多研究单位开展了阿秒科学与 技术研究, 代表性的单位有中国科学院西安光学精密机械研究所、中国科学院 物理研究所、中国科学院上海光学精密机械研究所及北京大学、华东师范大 学、华中科技大学、国防科技大学、吉林大学、西北师范大学、陕西师范大学 等, 并取得了一系列重要研究进展. 例如, 2013年, 中国科学院物理研究所在国内 首次产生了阿秒光脉冲 ${ }^{[2]}$. 此后, 中国科学院西安光学精密机械研究所、华中科 技大学、国防科技大学等也陆续获得了阿秒脉冲. 为了探讨我国阿秒光源前沿 科学和未来发展方向, 中国科学院西安光学精密机械研究所申请了以“阿秒光源 前沿科学与应用”为主题的第658次香山科学会议, 于2019年8月21 22日在北京 召开. 会议邀请中国科学院院士王恩哥、中国科学院西安光学精密机械研究所 赵卫研究员、中国科学院物理研究所魏志义研究员等担任执行主席, 来自国内 外 20 多家单位的 40 余名业内专家学者出席了会议. 与会专家围绕“阿秒光源国际 
发展趋势与我国机遇”和“阿秒实验技术及其在前沿科学领域中的应用”两个中心议题进行了深入讨论，形成了共 识并提出了重要建议. 为了在更大范围内共享本次香山科学会议的成果, 启迪创新思维和促进知识创新, 特组织 了本期专辑, 邀请了12位与会专家撰稿.

专辑包括阿秒光源前沿科学与技术研究领域的 12 篇文章 ${ }^{[3 \sim 14]}$, 内容涵盖阿秒光源产生原理和方案、驱动激光 研制进展、阿秒光源产生与测量技术，以及阿秒光源在基础物理和材料等多个前沿领域的应用进展．赵昆等人 ${ }^{[3]}$ 回顾了阿秒脉冲的测量方法和实验进展, 详细介绍了阿秒脉冲的时域信息重构算法; 兰鹏飞和陆培祥 ${ }^{[4]}$ 介绍了阿 秒脉冲的产生及其偏振、轨道角动量操控技术; 赵继民团队 ${ }^{[5]}$ 介绍了ATAS、atto-ARPES以及中红外超快光谱三 种实验技术及其在电介质、半导体、层状材料、过渡金属、超导体等材料中的应用; 曾志男团队 ${ }^{[6]}$ 介绍了使用相 对论强度激光在高密度等离子体中产生高强度阿秒光源的研究进展; 陈彦军团队 ${ }^{[7]}$ 介绍了利用高次谐波光谱重构 分子轨道、探测分子核动力学、标定分子取向等方面的研究进展; 付玉喜团队 ${ }^{[8]}$ 详细回顾了阿秒光源驱动激光的 发展现状, 并分析了其未来发展趋势; 魏志义团队 ${ }^{[9]}$ 综述了获得阿秒脉冲的原理、关键技术和发展历程; 刘运全团

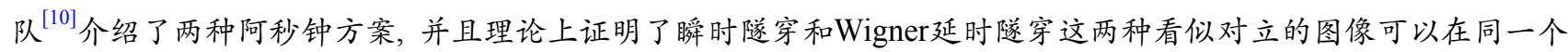
理论框架下进行描述; 赵增秀 ${ }^{[11]}$ 回顾了阿秒光源及相关技术在超快电子动力学测量和调控中的应用; 朱江峰团 队 ${ }^{[12]}$ 介绍了基于腔内共振增强、薄片/板条激光技术、相千合成等方法获得高重频驱动激光的研究进展; 柳晓军 团队 ${ }^{[13]}$ 使用玻姆力学方法深入研究了高次谐波类共振增强现象; 王国利团队 ${ }^{[14]}$ 开展了遗传算法优化多色激光场 波形来产生阿秒脉冲的理论研究.

衰心感谢各位专家为本专辑撰稿! 相信这些论文不仅会对国内工作于阿秒科学与技术研究领域的科研人员有促 进和指导作用, 也会为物理、化学、材料、信息、生物医学、能源等基础研究和应用基础研究领域的学者带来启发.

\section{参考文献}

1 Hentschel M, Kienberger R, Spielmann Ch, et al. Attosecond metrology. Nature, 2001, 49: 509-513

2 Zhan M J, Ye P, Teng H, et al. Generation and measurement of isolated 160-attosecond XUV laser pulses at 82 eV. Chin Phys Lett, 2013 , 30: 093201

3 Zhao K, Gao Y T, Zhu X X, et al. Principle and technology of attosecond pulse characterization (in Chinese). Chin Sci Bull, 2021, 66: 835-846 [赵 昆, 高亦谈, 朱孝先, 等. 阿秒脉冲测量原理和技术研究进展. 科学通报, 2021, 66: 835-846]

4 Lan P F, Lu P X. Generation and control of attosecond laser pulse (in Chinese). Chin Sci Bull, 2021, 66: 847-855 [兰鹏飞, 陆培祥. 阿秒激光脉冲 的产生与操控. 科学通报, 2021, 66: 847-855]

5 Hao W J, Zhai Y N, Zhang Q Y, et al. Attosecond light source in material science investigation (in Chinese). Chin Sci Bull, 2021, 66: 856-864 [郝 文杰, 翟燕妮, 张倩瑜, 等. 阿秒光源在材料领域的应用. 科学通报, 2021, 66: 856-864]

6 Wu J Q, Zeng Z N, Li R X. Theoretical and experimental progress of high-order harmonics and attosecond pulse generation based on solid overdense plasma (in Chinese). Chin Sci Bull, 2021, 66: 865-870 [吴家其, 曾志男, 李儒新. 基于固体等离子体的阿秒高次谐波产生理论与实 验进展. 科学通报, 2021, 66: 865-870]

7 Che J Y, Chen Y J. Advances on odd-even high-harmonic spectroscopy (in Chinese). Chin Sci Bull, 2021, 66: 871-877 [车佳殷, 陈彦军. 奇偶高 次谐波光谱学研究进展. 科学通报, 2021, 66: 871-877]

8 Yuan H, Cao H B, Wang H S, et al. Development and prospect on driving laser for attosecond pulse (in Chinese). Chin Sci Bull, 2021, 66: 878-888 [袁浩, 曹华保, 王虎山, 等. 阿秒脉冲驱动激光发展现状及展望. 科学通报, 2021, 66: 878-888]

9 Wei Z Y, Xu S Y, Jiang Y J, et al. Principle and progress of attosecond pulse generation (in Chinese). Chin Sci Bull, 2021, 66: 889-901 [魏志义, 许 思源, 江昱佼, 等. 阿秒脉冲产生的技术原理及进展. 科学通报, 2021, 66: 889-901]

10 Han M, Liu Y Q. Principle and application of two-color attoclock (in Chinese). Chin Sci Bull, 2021, 66: 902-912 [韩猛, 刘运全. 双色阿秒钟的原 理和应用. 科学通报, 2021, 66: 902-912]

11 Zhao Z X. Strong-field control and attosecond probing of multielectron dynamics (in Chinese). Chin Sci Bull, 2021, 66: 913-923 [赵增秀. 多电子 动力学的强场调控与阿秒探测. 科学通报, 2021, 66: 913-923]

12 Wang G Y, Lü R C, Xu S Y, et al. High repetition rate ultrafast laser technology for driving high-order harmonic generation (in Chinese). Chin Sci Bull, 2021, 66: 924-939 [王阁阳, 吕仁冲, 许思源, 等. 高重复频率高次谐波驱动源技术. 科学通报, 2021, 66: 924-939]

13 Lai X Y, Huang Y Y, Yu S G, et al. Interpretation of resonance-like enhancement in high-order harmonic generation with Bohmian mechanics (in Chinese). Chin Sci Bull, 2021, 66: 940-948 [赖炫扬, 黄祎神, 余少刚, 等. 高次谐波类共振增强的玻姆力学诠释. 科学通报, 2021, 66: 940-948]

14 Du J X, Wang G L, Jiao Z H, et al. Simulations on the single attosecond pulse generation by optimized multicolor laser field (in Chinese). Chin Sci Bull, 2021, 66: 949-956 [杜进旭, 王国利, 焦志宏, 等. 优化多色激光场产生单个阿秒脉冲的理论模拟. 科学通报, 2021, 66: 949-956] 